\title{
Modelo de formación en emprendimiento social para Instituciones de Educación Superior en Colombia
}

FECHA DE RECEPCIÓN: 27 de julio

FECHA DE APROBACIÓN: 26 de agosto pp. 29-44

DOI: http://dx.doi.org/10.21158/01208160.n81.2016.1563

\section{Resumen}

El artículo establece los componentes de un modelo de formación en emprendimiento social acorde con el contexto colombiano para lo cual se rastrean las investigaciones que permitan distinguir las particularidades que debe desarrollar el emprendedor social y establecer la formación que se está impartiendo en torno al emprendimiento, en particular, el caso de las Instituciones de Educación Superior (IES); adicionalmente, se realiza una aproximación al contexto de acción del emprendedor colombiano. Con los anteriores insumos se diseña una propuesta de modelo de formación en emprendimiento social que se aborda desde las dimensiones de: propósito, estrategia, entorno e individuo.

\section{Palabras clave}

Formación, emprendimiento social, emprendedor, instituciones de educación superior, modelo de formación.

Juan Camilo Vega Guerrero

Magíster en Administración de Empresas, Universidad Nacional de Colombia. Docente de la UNAD.

\section{Carlos William Mera Rodríguez}

PhD. (c) en Economía del Swiss Management Center University, Zurich-Suiza; Magíster en Educación, Pontificia Universidad Javeriana; especialista en Pedagogía y Docencia Universitaria, Universidad de San Buenaventura. Docente de la Universidad Nacional Abierta y a Distancia. 


\title{
Formation Model of Social Entrepreneurship for Higher Education Institutions in Colombia
}

\begin{abstract}
Abstrac. This article describes the components of a formation model for social entrepreneurship in accordance with the Colombian context, deeply analysing different research works to identify the special details that the social entrepreneur should develop, thus establishing the formation model that has been taught in regard with entrepreneurship, specifically the case of Colombian higher education institutions. Additional, an approximation to the context of the Colombian entrepreneur is made. Taking into account all these issues, a model for the development of social entrepreneurship is designed which includes the dimensions of purpose, strategy, context and individual.
\end{abstract}

Key words. Formation, social entrepreneurship, entrepreneur, higher education institutions, educational model.

\section{Modèle de formation à l'entrepreneuriat social des institutions d'éducation supérieure colombiennes.}

Resumé. Cet article établit les composants d'un modèle de formation entrepreneuriale dans le contexte social colombien. Les recherches sur ce sujet y sont regroupées pour permettre de distinguer les particularités que doit acquérir l'entrepreneur social afin d'établir la formation appropriée à ce type d'entrepreneuriat, principalement dans le cas des IES (Institutions d'Éducation Supérieure). Une analyse du contexte de l'entrepreneur colombien est par ailleurs réalisée. L'article proposera enfin un modèle de formation à l'entrepreneuriat social mis en place à partir d'objectifs, de stratégies et de l'environnement des individus.

Mots clefs. Formation, entrepreneuriat social, entrepreneur, Institution d'Education Supérieure, modèle de formation.

\section{Modelo de formação em empreendimento social, para instituições de ensino superior na Colômbia}

Resumo. O artigo tem o objetivo de estabelecer os componentes de um modelo de formação em empreendimento social em concordância com o contexto colombiano. Para isto, realiza-se um rastreamento sobre as pesquisas que permitem distinguir as particularidades a desenvolver pelo empreendedor social e também estabelecer como é a formação dada ao redor do empreendimento, e no caso especial nas IES (instituições de ensino superior). Adicionalmente, se realiza uma aproximação ao contexto de ação do empreendedor colombiano. Com todos os insumos sobreditos, traça-se una proposta de um modelo de formação em empreendimento social, o qual se aborda desde as dimensões de: propósito, estratégia, ambiente e individuo.

Palabras chave. Formação, empreendimento social, empreendedor, instituições de ensino superior, modelo de formação. 


\section{Introducción}

In Colombia, como en otros países lati1 noamericanos, se presentan altos niveles de actividad emprendedora y de iniciación de negocios, pero no se evidencian diferencias significativas entre las personas que recibieron formación empresarial frente a quienes no la tienen (Gómez et al., 2011). Así mismo, los programas de formación en administración de empresas tienen falencias en la promoción de la innovación, la creatividad y el trabajo por cuenta propia, los cuales son aspectos fundamentales en la educación hacia el emprendimiento (Comisión Europea, 2009). Conceptualmente, se reconocen dos tipos de emprendimiento: el de negocios y el social. El primero, orienta su propósito en obtener una remuneración económica, y el segundo, actúa para dar respuesta a una problemática en la sociedad (Guzmán y Trujillo, 2008). Es de destacar, la importancia del emprendimiento social para el desarrollo económico en países subdesarrollados o emergentes (Guzmán y Trujillo, 2008), como también la relevancia de este tipo de emprendimiento en la generación efectiva de puestos de trabajo, según la investigación realizada por el Observatorio Global del Reino Unido en 2003 (Harding, 2004). De allí, la relevancia de la investigación en temas de formación en emprendimiento social, y por lo cual son las Instituciones de Educación Superior las responsables de dinamizar los programas de formación en emprendimiento, como también que se conviertan en aliados estratégicos para los emprendedores que quieran poner en marcha sus negocios o empresas (Arias y Giraldo, 2011).
La investigación explora los aspectos que deberían abordar los programas de formación en administración de empresas y negocios orientados al emprendimiento social, en Instituciones de Educación Superior en Colombia, y que además den respuesta al siguiente interrogante: ¿cuáles son las dimensiones ${ }^{1}$ que debería abordar un programa de formación en emprendimiento social en las IES en Colombia? En consecuencia, la investigación tiene como objetivo caracterizar las dimensiones que inciden en la formación de individuos en emprendimiento social para las IES en Colombia, razón por la cual se realiza un análisis de contenidos en documentos e investigaciones sobre emprendimiento social a nivel general, y en el contexto colombiano. Finalmente, se diseña una propuesta de modelo en emprendimiento social destacando las siguientes características:

- Articulación de la práctica en los espacios de aprendizaje para el desarrollo de competencias ciudadanas y en gestión organizacional.

- Implementar el aprendizaje hermenéuticoreflexivo como herramienta para la resolución de problemas reales en la comunidad.

- Incentivar en el individuo la capacidad relacional y el trabajo en equipo.

\footnotetext{
${ }^{1}$ Cada una de las magnitudes de un conjunto que sirve para definir un fenómeno. Definición tomada del diccionario de la Real Academia Española, disponible en: www.rae.es.
} 


\section{Metodología}

I estudio es de carácter exploratorio y aplica el método de análisis de contenidos

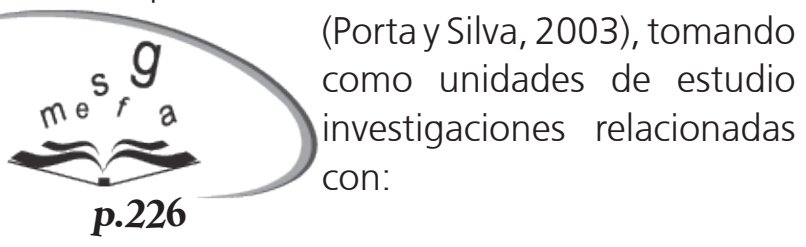

- Emprendimiento social, específicamente en el contexto colombiano.
- Perfiles de formación en emprendimiento desarrollados en programas de administración de empresas y negocios.

Finalmente, se realiza la categorización y análisis de las unidades de estudio para diseñar la propuesta de modelo de formación en emprendimiento social.

\section{Revisión de literatura}

\subsection{El emprendedor social y sus características}

Una primera aproximación a la definición de emprendedor lo identifica como la persona que «emprende y se determina a hacer y ejecutar con resolución y empeño alguna operación considerable y ardua», según la Real Academia de la Lengua Española (1791). En este sentido, es desde la economía que inicialmente se aborda el tema de emprendimiento; al respecto Schumpeter $(1961,1965)$ complementa la definición mencionada enfatizando en el propósito económico que busca el emprendedor, y adicionalmente, denominándolo como destructor creativo, quien rompe los ciclos de mercado y lleva a reducir los precios del producto para lograr un rendimiento financiero sobre el capital invertido mediante la introducción de una innovación que le brinda una ventaja en el tiempo. En esta misma corriente de pensamiento, Cantillon (1755) asocia al emprendedor como una persona que compra productos a precios conocidos para venderlos a precios desconocidos en condiciones de incertidumbre, por lo que asume riesgos y busca ganancias variables e inciertas. Otro aporte interesante para la comprensión del concepto es la de Baptiste (1767-1832), quien enuncia que el individuo emprendedor tiene la capacidad de integrar los medios de producción para construir un ente productivo. Así mismo, Liouville (2002) adiciona un elemento interesante a las características del emprendedor: «la de tomar iniciativas de crear».

De igual manera, otra mirada interesante del individuo emprendedor parte de las ciencias humanas relacionadas con su personalidad, distinguiéndose entre otras las siguientes:

- Tomador de riesgos (Kirby, 2004).

- Con orientación al logro (Timmons \& Spinelli, 2007).

- Capacidad de trabajo en equipo (Sarasvath, 2001).

- Facilidad de adaptación al cambio (Gibb, 2005). 
Otros elementos relevantes de la caracterización del emprendedor parten desde la disciplina administrativa y giran en torno a los procesos estratégicos y de gestión; en este sentido, se recalca la capacidad del emprendedor para identificar e interpretar oportunidades, así como la de explotar de manera organizada la oportunidad identificada (Busenitz et al., 2003). Pereira (2003), define las características del emprendedor sobre tres ejes:
- El entorno².

- Las características individuales del emprendedor ${ }^{3}$.

- Los procesos emprendedores ${ }^{4}$.

De otro lado, Stevenson (1983) categoriza las dimensiones organizacionales que debe gestionar el emprendedor y caracteriza los retos que este debe asumir. (Tabla 1).

Tabla 1. Cultura del espíritu emprendedor.

\begin{tabular}{|c|l|l|}
\hline $\begin{array}{c}\text { Orientación } \\
\text { estratégica }\end{array}$ & $\begin{array}{l}\text { Impulsada por la } \\
\text { percepción de la } \\
\text { oportunidad. }\end{array}$ & $\begin{array}{l}\text { - Disminución de las oportunidades. } \\
\text { - Tecnología rápidamente cambiante, situación } \\
\text { económica de los consumidores, valores sociales y normas } \\
\text { políticas. }\end{array}$ \\
\hline $\begin{array}{c}\text { Compromiso } \\
\text { con el } \\
\text { de oportunidades }\end{array}$ & $\begin{array}{l}\text { Revolucionario, de corta } \\
\text { duración. }\end{array}$ & $\begin{array}{l}\text { - Orientación a la acción. } \\
\text { - Ventanas de decisión estrechas. } \\
\text { - Aceptación de riesgos razonables. } \\
\text { - Los grupos que participan en las decisiones son pocos. }\end{array}$ \\
\hline $\begin{array}{c}\text { Compromiso de } \\
\text { recursos }\end{array}$ & $\begin{array}{l}\text { Muchas etapas, con } \\
\text { exposición mínima en } \\
\text { cada etapa. }\end{array}$ & $\begin{array}{l}\text { - Falta de necesidades predecibles de recursos. } \\
\text { - Falta de control sobre el entorno. } \\
\text { - Exigencias sociales de uso adecuado de recursos. } \\
\text { - Competencia extranjera. } \\
\text { - Exigencias de un uso más eficiente de los recursos. }\end{array}$ \\
\hline $\begin{array}{c}\text { Control de } \\
\text { recursos }\end{array}$ & $\begin{array}{l}\text { Uso en etapas o } \\
\text { arriendo de los recursos } \\
\text { necesarios. }\end{array}$ & $\begin{array}{l}\text { - Mayor especialización de los recursos. } \\
\text { - Larga vida de los recursos en comparación con la necesidad. } \\
\text { - Riesgo de obsolescencia. } \\
\text { - Riesgo inherente a la oportunidad indentificada. } \\
\text { - Inflexibilidad del permanente compromiso con los recursos. }\end{array}$ \\
\hline $\begin{array}{c}\text { Estructura } \\
\text { generencial }\end{array}$ & $\begin{array}{l}\text { Plana, con múltiples } \\
\text { redes informales. }\end{array}$ & $\begin{array}{l}\text { - Coordinación de recursos claves no controlados. } \\
\text { - Desafío a la jerarquía. } \\
\text { - Deseo de independencia de los empleados. }\end{array}$ \\
\hline
\end{tabular}

Fuente. Stevenson, (1983).

\footnotetext{
2 Entendido como el contexto económico, y como las condiciones estructurales que influyen en todos los actores económicos de un país.

3 Incluye las habilidades aptitudinales, los comportamientos actitudinales del emprendedor.

4 Proceso emprendedor: "Conjunto de las funciones, actividades y acciones asociadas con la percepción de la oportunidad y la creación de la organización para explotarla» (Bygrave y Hofer, 1991).
} 
Basado en lo anterior, las características básicas del emprendedor se pueden categorizar en: aptitudinales, relacionadas con el uso efectivo de estrategias, metodologías y herramientas de gestión; y actitudinales, enfocadas en la determinación para la búsqueda de oportunidades de negocio, el desarrollo innovador de las ideas de negocio y la disposición para asumir riesgos. También, se identifican dos escenarios de acción del emprendedor: la organización, relacionada con el espacio donde el emprendedor acopla sus concepciones éticas ${ }^{5}$ y axiológicas, con las del grupo humano de trabajo para el proceso de toma de decisiones; y el entorno, entendido como el contexto donde el emprendedor implementa estrategias e ideas de negocio.

\section{La ética es la filosofía que reflexiona sobre la moral, y pretende legitimarla, o des- legitimarla, a partir de unos principios éticos. Estos últimos son universales, ya que deben ser compartidos y respetados por cualquier individuo, independiente- mente de sus valores, de su moral. De ahí que también se defina a la ética como el conjunto de principios y reglas morales que regulan el comportamiento y las relaciones humanas (Agulló, 1990).}

Bajo estos parámetros, es relevante focalizar la compresión de las características y de los entornos mencionados para el caso del emprendedor social.

El emprendedor social es un tipo de emprendedor que persigue fines sociales (Curto, 2012), en consecuencia, el principal aspecto que lo define es su compromiso con generar impacto social, entendido este como «un mejoramiento significativo, en algunos casos, perdurable o sustentable en el tiempo, en alguna de las condiciones o características de la población objetivo y que se plantearon como esenciales en la definición del problema que dio origen» y cuyo «resultado final suele expresarse como un beneficio a mediano y largo plazo obtenido por la población atendida» (Guzmán, 2004). Los principales impactos sociales que persiguen este tipo de emprendedores son:

- Cubrir necesidades de un colectivo determinado (Melián, Camps y Sanchis, 2011).

- Generar puestos de trabajo (Melián, Camps y Sanchis, 2011).

Es de destacar que los emprendedores sociales son individuos idealistas con fuertes convicciones éticas, religiosas y morales (Melián, Camps y Sanchis, 2011), y cuyo perfil de gestión se enfatiza en:

- Fortalecer las relaciones entre los participantes de la organización (Chaves y Sajardo, 2004; Gómez, 2003; Kliksberg, 2004; Moulden, 2009; Zandonai y Pezzini, 2004).

- La dirección participativa, haciendo énfasis en el contacto directo entre el empresario y las comunidades hacia las que se dirige la organización (Chaves y Sajardo, 2004).

- La conformación de estructuras organizaciones flexibles -horizontales- (Melián, Campos y Sanchis, 2011).

- Capacidad para difundir las innovaciones sociales en el mayor número poblacional posible (Dees, J. G., Anderson, B. B. \& WeiSkillern, J., 2004).

- Las alianzas, las buenas relaciones con los clientes y las instituciones gubernamentales (Melián, Campos y Sanchis, 2011).

- El aprendizaje continuo de los individuos (Curto, 2012). 
- La innovación como eje fundamental para que grupos marginados utilicen y maximicen los activos de los que disponen (Curto, 2012).

Por otro lado, el accionar del emprendedor social se desenvuelve dentro de los contextos, principalmente, de tres aspectos (Curto, 2012):

- Dotar a la comunidad con los recursos para solucionar problemas particulares.

- Proporcionar instrumentos para solucionar problemas comunes.

- Organizar movimientos locales para hacer frente a actores más poderosos.

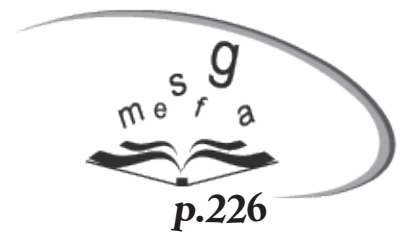

En consecuencia con el alcance de la propuesta, es necesario identificar el perfil del emprendedor colombiano. Al respecto, se pueden identificar dos aspectos que motivan a este tipo de individuos en Colombia:

- La movilidad social5 (Gómez, 1999).

- La cultura rentista6 (Garay, 1999).

Con respecto a las características aptitudinales y actitudinales de los emprendedores en Colombia, se distinguen rasgos particulares en lo referente a innovación7, administración ${ }^{8}$ y toma de riesgo $^{9}$ (Kets de Vries, 1977), y se relacionan a continuación:

En innovación:

- Alta capacidad creativa individual (Pereira, 2003).

- Una pobre conciencia de participación e integración colectiva (Pereira, 2003).

\section{En administración:}

- Marcado individualismo, lo que dificulta el trabajo en equipo (Gómez, 1999).

En toma de riesgo:

- Asume riesgos difícilmente calculables debido a la complejidad del entorno donde se desenvuelve (Pereira, 2003).

- El emprendedor se involucra en la gestión del Estado para influir en la toma de decisiones estatales y lograr beneficios particulares.

- Diversificación de las inversiones con el fin de protegerse de un entorno inestable, lo que ocasiona una baja especialización (Dávila, 1997).

- Predomina la toma de decisiones donde prima el interés particular sobre el colectivo.

\footnotetext{
5 Sociedad que aplaude los éxitos individuales y son factor de reconocimiento y éxito.

6 Priman los intereses particulares.

7 Función del emprendedor relacionada con una combinación novedosa de factores de producción existentes. Para conocer más sobre el tema, consultar: Shumpeter, (1965).

8 La consecución de nuevas fuentes de recursos, métodos de mercadeo y estructura de la organización. Para conocer más sobre el tema, consultar: Shumpeter, (1965).

9 En este aspecto, el emprendedor arriesga su capital financiero, su imagen social y los costos psicológicos asociados con el fracaso. Para ampliar este aspecto, consultar: Pereira (2003).
} 
Finalmente, el entorno, según Pereira (2003), donde se desenvuelve el emprendedor colombiano tiene una marcada falencia en lo concerniente a:

- Falta de capital de riesgo para crear empresa (Global Competitiveness Report, 2001).

- Los recursos para el acceso al crédito se soportan sobre garantías financieras y no por planes de negocio viables (Global Competitiveness Report, 2001).

- Baja inversión en investigación (Global Competitiveness Report, 2001).

En este punto se presenta una aproximación al tipo de formación que imparten las IES.

\subsection{La formación en emprendimiento}

La universidad no es ajena al propósito sobre capacitación en emprendimiento. Al respecto, Kirby (2004), identifica tres categorías de programas de formación en emprendimiento:

- Orientados a brindar información sobre emprendimiento y motivar una actitud positiva hacia este.

- Promueven la creación de nuevas empresas que fomentan el autoempleo, la autosuficiencia económica y la generación de empleo.
- Direccionados al crecimiento de la pequeña empresa.

La discusión de formación en emprendimiento tiene múltiples miradas, por ejemplo:

- El abordaje de los conocimientos prácticos y de gestión para el desarrollo del emprendimiento (Vesper, 1985).

- El conocimiento disciplinar, que comprende los aspectos teóricos y empíricos de las disciplinas que propenden por entender el ámbito del emprendedor, que involucra los planteamientos desde las ciencias económicas, de la organización y humanas (Bechard y Toulouse, 1998).

- El epistemológico, que pretende caracterizar el emprendimiento de forma integral (Bechard y Toulouse, 1998).

Un aporte importante en el tema es el de Gorgan (1997), quien identifica tres tipos de educación en emprendimiento según sus objetivos: la enfocada en generar el espíritu emprendedor, la empresarial y la de gestión de pequeñas empresas; así mismo, determina que los componentes de la estructura en formación en emprendimiento se centran en los siguientes aspectos:

- El desarrollo de capacidades.

- En experiencias obtenidas en proyectos.

- En las tareas.

- En contenidos que influyan en el desarrollo del espíritu emprendedor ${ }^{10}$.

\footnotetext{
${ }^{10}$ Este componente de formación presenta la información general sobre emprendimiento y plantea reflexiones sobre la carrera en emprendimiento.
} 
A grandes rasgos, se evidencia que existe un abordaje de la enseñanza del emprendimiento de forma fragmentada y que su desarrollo ha sido de forma lenta (Gibb, 2005). Por ello, desde la universidad es pertinente diseñar e implementar un modelo de formación del emprendimiento que motive al estudiante a tomar e implementar acciones emprendedoras.

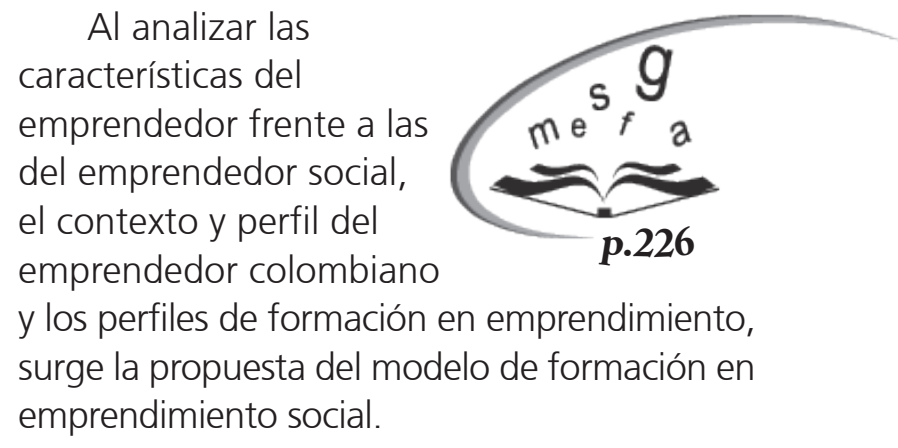

Al analizar las características del emprendedor frente a las del emprendedor social, el contexto y perfil del y

\section{Resultados}

\subsection{Modelo de formación para el emprendimiento en Colombia}

Se plantea un modelo ${ }^{11}$ de formación en emprendimiento social que consta de tres dimensiones en confluencia con el individuo (Figura 1):

- Los propósitos: esta dimensión agrupa y direcciona las competencias ${ }^{12}$ de formación en emprendimiento acorde con las características propias del emprendedor social y acopladas al entorno colombiano; en este sentido, los propósitos de formación deben focalizarse en desarrollar:

- Características actitudinales: relacionada con las normas, valores y perfiles de conducta con respecto a la interacción con el contexto y los distintos grupos de interés. Así se destacan: a. El liderazgo participativo y de trabajo en equipo.

b. La búsqueda y acercamiento con comunidades vulnerables para identificación de problemáticas sociales.

c. La ética del servicio y la responsabilidad social13: orientada a establecer unos principios de conducta básicos que medien en las relaciones y la toma de decisiones con respecto a los grupos de interés con los que interactúa y que faciliten el trabajo en equipo y la consecución de objetivos ${ }^{14}$.

d. La búsqueda del reconocimiento del trabajo del grupo y no del individual.

e. La satisfacción con el trabajo traducida en logros e impactos en la comunidad y no en términos monetarios.

11 Es importante mencionar, que los modelos son analogías para entender la realidad, que incluyen un conjunto de afirmaciones o elementos, y cuya validación parte del análisis lógico de cada modelo y de sus relaciones con las evidencias observables del fenómeno que son el soporte de este (Caldin, 2002; Gallego, 2004).

12 En cuanto a lo mencionado, se trata del desarrollo de capacidades complejas que permiten a los estudiantes pensar y actuar en diversos ámbitos. Consiste en la adquisición a través de la acción, resultado de una cultura de base sólida que puede ponerse en práctica y utilizarse para explicar lo que está sucediendo, (Unesco, 2013).

13 En torno a este tema, la responsabilidad social es la contribución voluntaria que hacen las organizaciones para incrementar al máximo los beneficios de los distintos grupos de interés, procurando la sostenibilidad de la organización, el buen gobierno, minimizando el daño ambiental y satisfaciendo las necesidades de la sociedad (Riaño, 2015).

14 En este aspecto, debe dirigir el comportamiento del emprendedor colombiano para mitigar el uso de prácticas emprendedoras asociadas con la corrupción y la manipulación de los entes gubernamentales o de sus representantes. 
- Características aptitudinales -el saber hacer-: orientadas a dar las herramientas y habilidades para identificar y gestionar oportunidades para el desarrollo de proyectos sociales dentro de su entorno; estas se encuentran focalizadas en:

a. Fomentar las habilidades y competencias comunicativas en el idioma nativo y en una segunda lengua para mejorar la capacidad relacional.

b. Desarrollar capacidades para el diseño de proyectos sostenibles y el manejo de herramientas de gestión para elaborar proyectos sociales con el fin de acceder a recursos de entidades estatales $u$ organizaciones que apoyen este tipo de iniciativas.

c. Manejo de herramientas telemáticas y comunicativas.

d. Capacidad de estructurar organizacionales flexibles.

- La estrategia de formación: entendida como la ruta de apropiación de los propósitos de formación en emprendimiento social, para el caso en particular de formación, se propone una orientada sobre los modelos de aprendizaje ${ }^{15}$ hermenéutico-reflexivo (Pérez, 1996), la cual se soporta sobre:

a. Ecosistemas inestables.

b. Sobredeterminada por contextos -espaciales, temporales y sociopolíticos-.

c. Cargada de conflictos de valor que requieren alternativas éticas y políticas.
Este tipo de modelo de enseñanza se asemeja a las características del entorno al cual se enfrentaría el emprendedor social, particularmente, el colombiano. Este modelo permite:

a. La construcción personal y colectiva de conocimiento.

b. Establecer parte de las situaciones concretas.

c. Utilizar la reflexión para entender las situaciones, y usar la práctica para volver a la situación y modificarla.

d. La generación de nuevo conocimiento

e. La práctica, que es su eje estructural.

- El entorno: entendido como el espacio próximo al individuo donde este forma sus expectativas y creencias (Gibb, 2005), y de donde identifica oportunidades para desarrollar e implementar proyectos sociales; aplica sus capacidades aptitudinales, enmarcadas dentro de los preceptos actitudinales adquiridos. Al respecto, debido a la baja especialización de los individuos emprendedores en el mercado y asociado con la falta de capital o acceso para crear empresa, es relevante que los programas de formación se focalicen en generar propuestas de investigación e innovación que busquen integrar grupos marginados con entidades privadas o gubernamentales.

- Individuo: es la conjunción de atributos personales, como estados afectivos, cognitivos, físicosy biológicos, yotros comportamentales

\footnotetext{
${ }^{15}$ Al respecto de este término, se puede ampliar su definición a partir de Gallego et. al. (2004): en cuanto a que el «aprendizaje es el proceso de adquisición de una disposición, relativamente duradera, para cambiar la percepción o la conducta como resultado de una experiencia»
} 
(Bandura, 1986). En este sentido se puede establecer que el emprendedor colombiano orienta sus esfuerzos en obtener beneficios rentistas basados en el trabajo individual.

De esta manera, se pueden describir las relaciones de cada uno de los elementos:

- Propósitos-estrategia: definen las competencias y valores en emprendimiento social que deben ser transferidos al individuo para desarrollar proyectos sociales.

- Estrategia-individuo: desarrolla la metodología y herramientas de aprendizaje para que el individuo reflexione, comprenda el contexto e identifique problemáticas sociales.

Implementa escenarios para que el estudiante analice y tome decisiones para desarrollar proyectos sociales que den solución a problemas en la comunidad o en grupos vulnerables.
Diseña las mediaciones articuladas con la práctica y la realidad para que el individuo interactúe con el contexto.

- Individuo-entorno. Esta relación permite que el individuo:

- Intervenga las comunidades para buscar conjuntamente soluciones a las problemáticas sociales.

- Se integre o genere proyectos sociales de extensión, investigación o innovación, para ser patrocinada por entidades gubernamentales o privadas.

- Entorno-propósito: modifica los propósitos de formación basado en las necesidades de la comunidad, las formas como se relacionan los individuos y las comunidades, y los comportamientos de conducta establecidos por la sociedad.

Figura 1. Modelo de formación para el emprendimiento en Colombia.

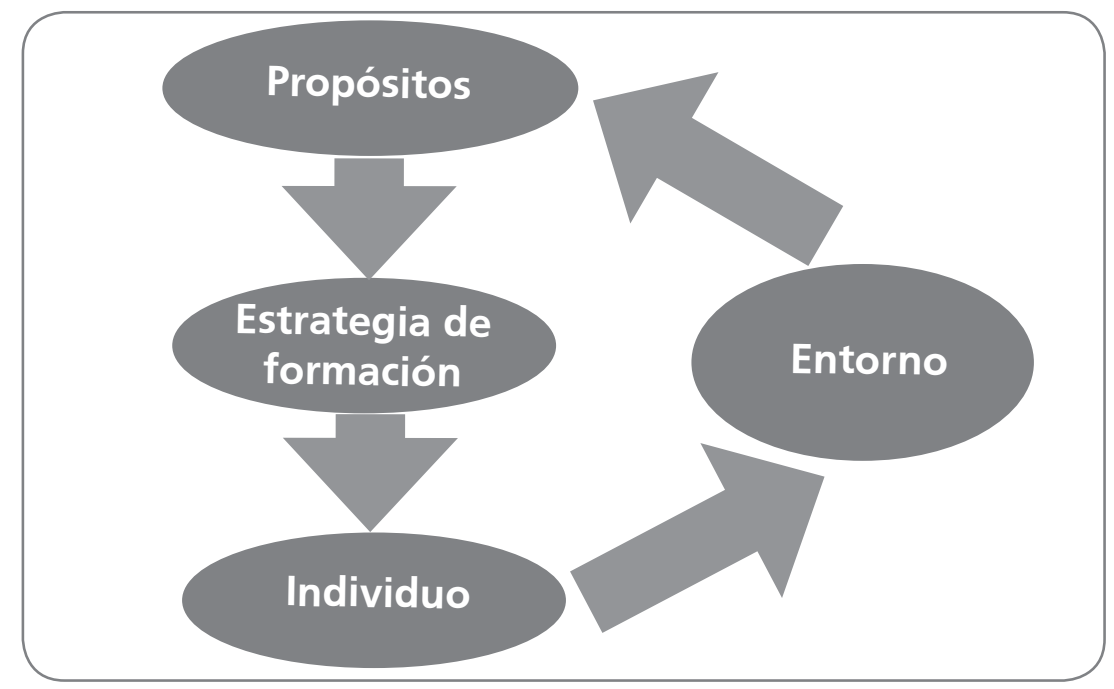

Fuente. Elaboración propia de los autores. 


\section{Conclusiones}

L as principales dificultades que tiene el desarrollo de programas de formación en emprendimiento social recaen en dos aristas: la primera, relacionada con el individuo, debido a la marcada tendencia del emprendedor colombiano de trabajar de forma individual y buscando logros económicos o de movilidad social, los cuales son preceptos que van en contravía alos fines teleológicos deformaciónen emprendiendo social. La segunda, enmarcada en los programas de formación en administración de empresas que hacen énfasis en los contenidos y en las competencias que debe desarrollar el emprendedor -relacionadas con oportunidades de negocio-. Adicionalmente, los programas adolecen de una estrategia de aprendizaje y de pedagogía pertinente para la formación en emprendimiento social.

En consecuencia, el modelo de formación en emprendimiento social toma como eje fundamental al individuo, y reconoce como primera dimensión, qué incide en su proceso de aprendizaje, además de los propósitos que persigue, destacando como perfil de interés la importancia para el emprendedor social desarrollar la capacidad relacional y el trabajo en equipo, que le permiten:

- Intervenir en la comunidad para solucionar problemáticas sociales del contexto (Chaves y Sajardo, 2004).

- Diseñar equipos de trabajo participativos (Chaves y Sajardo, 2004; Gómez, 2003; Kliksberg, 2004; Moulden, 2009; Zandonai y Pezzini, 2004).
- Articular diferentes organizaciones estatales y particulares para el financiamiento de proyectos sociales (Melián, Campos y Sanchis, 2011).

La segunda dimensión del modelo de formación en emprendimiento social es la de estrategia de formación, enfatizando que esta se debe fundamentar en un proceso de aprendizaje hermenéutico-reflexivo (Pérez, 1996), donde prima:

- El desarrollo de actividades a partir de problemáticas reales del contexto.

- La intervención en el contexto, fundamentada en la práctica.

- La construcción colectiva del conocimiento.

- La toma de decisiones éticas y responsables en escenarios conflictivos.

Finalmente, la tercera dimensión que incide en la formación en emprendimiento social es el entorno, entendido como el contexto donde el individuo interactúa y donde desarrolla proyectos para mitigar o solucionar problemáticas sociales. Al respecto se identifican obstáculos relacionados con las fuentes de financiación, baja inversión en investigación, y prácticas de negocio que buscan influir en la toma de decisiones de órganos de gobierno para el beneficio particular. 


\section{Referencias bibliográficas}

Arias, M., y Giraldo, D. (2011). Condiciones para la generación de emprendimientos en Medellín y su área metropolitana. Revista Virtual Universidad Católica del Norte, Medellín, Colombia. Recuperado de: http://www.redalyc.org/articulo.oa?id= 194222473007

Barbe, W., \& Swassing, R. (1979). Teaching through Modality Strengths: Concepts and Practices. Ohio: Zaner-Bloser Inc.

Bechard, J., \& Toulouse, J. (1998). Validation of a didactic model for the analysis of training objectives in entrepreneurship. Journal of Business Venturing.

Busenitz, L., West, G., Shepherd, D., Nelson, T., Chandler, G., \& Zacharakis, A. (2003). Entrepreneurship in emergence: Fourteen years of entrepreneurship research in manaGEMent journals. Journal of ManaGEMent.

Caldin, E. (2002). The Structure of Chemistry in Relation to the Philosophy of Science. HYLE. International Journal for Philosophy of Chemistry, 8 (2), 103-121. Recuperado de: http://hyle.org/journal/issues/8-2/caldin. html.

Cantillon, R. (1755). Premiére partie en Essai sur la natures du commerce en general. Londres: Mac Millán. (Editado en 1931).

Castellanos, F., \& Fonseca, S., et al. (2013). Requerimientos del sector productivo colombiano: la administración del siglo XXI. Vicerrectoría de Investigación, Universidad Nacional de Colombia.
Chaves, R., \& Sajardo, A. (2004). Economía política de los directivos de las empresas de economía social. CIRIEC-España, 48, pp. 31-52.

Curto, M. (2012). Los emprendedores sociales: innovación al servicio del cambio social. Cuadernos de la Cátedra la Caixa de Responsabilidad Social de la Empresa y Gobierno Corporativo, 13. IESE Business School, Universidad de Navarra.

Dávila, C. (1997). Historia empresarial de Colombia: estudios, problemas y perspectivas. Monografía, 20. Serie Historia Empresarial, segunda reimpresión, febrero 1997. Bogotá: Universidad de los Andes, Facultad de Administración.

Dees, J., Anderson, B., \& J. Wei-Skillern. (2004). Scaling Social Impact, Stanford Social Innovation Review, 1(4), pp. 24-32.

Fonseca, H. (2013). Estilos de enseñanza y aprendizaje. Primer Congreso Internacional de Pedagogía, Didáctica y Tecnologías de la Educación. Bogotá, Colombia. Universidad autónoma de Colombia.

Gallego, R. (2004). Un concepto epistemológico de modelo para la didáctica de las ciencias experimentales. Revista electrónica de enseñanza de las Ciencias 3 (3) pp.301-319. Recuperado de: http:// www.saum.uvigo.es/reec/volumenes/ volumen3/Numero3/ART4_VOL3_N3.pdf

Gibb, A. (2005). Towards the Entrepreneurial University Entrepreneurship Education as a lever for change. NCGE Policy paper series. 
Gómez, L., Martínez, P., Figueroa, C., Pereira, F., Quiroga, R., Vesga, R., Varela, R., y Álvarez, L. (2011). Reporte GEM Colombia 2010. Editorial Universidad del Norte.

Gorman, G., Hanlon, D., \& King, W. (1997). Home research perspectives on entrepreneurship education, enterprise education and education for small business management: A ten-year literature review. International Small Business Journal, 15 (3), pp. 56-77.

Guzmán M. (2004). Metodología de evaluación de impacto. Santiago de Chile: División de Control de Gestión. Recuperado de: http://hidroven.gov. ve/Resultados/Evaluaci \% C3\%B3n \% 20 de\%20Impacto\%20Gesti\%C3\%B3n.pdf

Guzmán y Trujillo, (2008). Emprendimiento Social. Revisión de Literatura Estudios Gerenciales 24 (109), Universidad ICESI, Cali, Colombia

Kets de Vries, M. (1977). The Entrepreneurial Personality - A Person at The Cross -roads. Journal of Management Studies, 14 (1), pp. 34-47.

Kirby, D. (2004). Entrepreneurship education: can business school meet the challenge? Education + Training.

Kliksberg, B. (2004). La ética y el capital social cuentan. Universidad de Carabobo (UCAB), Ediciones de la FCES de la UCAB. Venezuela.

Kolb, D. (1984). Experiential learning. Experience as the source of learning and development. New Jersey, Estados Unidos. Prentice Hall.
Liouville, J. (2002). La fonction d'entrepreneur: Schumpeter revisité. Académie de I' Entrepreneuriat, Les actes du 2e congrès, Bordeaux, France.

Melián, A., Campos, V., \& Sanchis, J. (2011). Emprendimiento social y empresas de inserción en España. Aplicación del método Delphi para la determinación del perfil del emprendedor y las empresas sociales creadas por emprendedores. Revesco, 106, ISSN: 1885-8031. DOI:1.5209/rev_ REVE.2011.v106.37377.

Moulden, J. (2009). Los nuevos emprendedores sociales. Ed. McGraw-Hill. México.

Pereira, F. (2003). Reflexión sobre algunas características del espíritu emprendedor colombiano. Revista Economía, Gestión y Desarrollo, Cali, Colombia.

Pérez, A. (1996). Autonomía profesional del docente y control democrático. En varios autores, Volver a pensar la educación. Morata. Madrid, España.

Porta, L., \& Silva, M. (2003). La investigación cualitativa: el análisis de contenido e $n$ la investigación educativa. Universidad Nacional de Mar del Plata, Universidad Nacional de la Patagonia Austral. Recuperado de: http://www.uccor.edu.ar/ paginas/REDUC/porta.pdf

Real Academia Española. (1791). Diccionario de la Real Academia Española. Madrid, España. Imprenta de la Real Academia Española.

Real Academia Española. (1837). Diccionario de la Lengua Castellana de la Real Academia Española. Madrid, España, Imprenta de la Real Academia Española. 
Schumpeter, J. (1965). Economic Theory and Entrepreneurial History en H.C. Aitken, Exploratins in Enterprise. Cambridge, Harvard University Press.

Silva, G. (1990). La coherencia ética en la gestión de los recursos humanos: Un factor clave para la forja del ethos corporativo. (Tesis doctoral). Universidad Ramon Llull, Barcelona, España. Recuperado de: http://www.tesisenred.net/bitstream/ handle/10803/9317/Tesis_Silvia_Agullo. pdf? sequence $=1$

Stevenson, H. (1983). A Perspective on Entrepreneurship. Harvard Business School Working, 9, p. 384-131.

Timmons, J., \& Spinelli, S. (2007). New Venture Creation. Boston: McGraw-Hill.
Unesco. (2013). Enfoque por competencias. Oficina Internacional de Educación. Recuperado de: http:www.ibe.unesco.org/ es/comunidades/comunidad-de-practicacop/enfoque-por-competecias.html.

Vesper, K. (1985). A new direction, or just a new label?, in J. J. Kao and H. H. Stevenson (eds), Entrepreneurship: What It Is And How To Teach It, Boston, MA: Harvard Business School,

Zandonai, F., \& Pezzini, E. Nuevos directivos para nuevas cooperativas. La figurad e I empresario social en las cooperativas sociales italianas, Revista CIRIECEspaña, (48), p. 145-166. 
